\title{
Scrutiny of Breast Cancer Detection Techniques of Deeplearning and Machine Learning
}

\author{
Kiranpreet Kaur, S.K. Mittal
}

\begin{abstract}
Breast cancer is one of the most widely recognized tumors globally among ladies with the data available that one of every eight ladies is influenced by this illness during their lifetime. Mammography is the best imaging methodology for early location of the disease in beginning times. On account of poor complexity and low perceivability in the mammographic pictures, early discovery of the cancer malignant growth is a huge challenge to effective cure of the disease. Distinctive CAD (computer aided detection) supported algorithms have been developed to enable radiologists to give an exact determination. This paper highlights the study of the most widely recognized methodologies of image segmentation created for recognition of calcifications and masses. The principle focal point of this survey is on picture theof strategies and the factors utilized for early bosom disease identification. Surface investigation is the vital advance in any picture division strategies of image segmentation which depend on a nearby spatial variety of color or shading. Subsequently, different techniques for texture investigation for small scale calcification and mass identification in mammography are talked about in the mechanism of mammography. The point of this paper is to audit existing ways to deal with the segmentation of masses and automated detection in mammographic pictures, underlining the key-focuses and primary contrasts among the utilized systems. The key goal is to bring up the preferences and drawbacks of the different methodologies. Conversely with different surveys which just portray and think about various methodologies subjectively, this audit likewise gives a quantifiable examination.In proposed research use deep learning base network for classification of mammography images. In previous approaches use machine learning base learning. The Main drawback of machine learning is selection of features manualy or by functions but in deep learning automatic feature detect and its vary according to image. The demonstration of seven mass recognition techniques is thought about utilizing two distinctive databases of mammography: an open digitized database and a full-field (local) advanced digitized database. The outcomes are given as far as Free reaction Receiver Operating Characteristic (FROC) and Receiver Operating Characteristic (ROC) examination.
\end{abstract}

Keywords : Computer aided design, Convolutional neural networks, Deep learning, Mammography.

\section{INTRODUCTION}

Ongoing investigations have demonstrated that the growth of breast cancer is the most widely recognized kind of disease among women [33], marking around $33 \%$ of recently analyzed tumors in the US [19, 36]. The death rate of breastcancer is likewise high, representing $17 \%$ of passings identified with disease in general [31]. Exact detection and

Revised Manuscript Received on September 25, 2019

Kiranpreet Kaur, Research Scholar, Department of Computer Science and enggenring, Rayat Bahra University,Sahauran, Punjab

Dr. S.K. Mittal, Professor, Department of Computer Science and enggenring, Rayat Bahra University,Sahauran, Punjab evaluation of the disease in its beginning times is critical with regards to diminishing the rate of mortality. Currently mammography until know is considered as the most valuable apparatus for all inclusive community screening. Though, the precise discovery and conclusion of a breast injury or lesion is exclusively dependent on mammography discoveries is troublesome and highly relies upon the ability of the radiologist, which prompts a great number of false positives and spareinvestigations $[55,57]$. The computer-aided detection and diagnosis specifically known as CAD frameworks are now being utilized to offer or suggestvital support in the process of radiologists based decision-making. Such frameworks may essentially lessen the needed effort required for the evaluation of a lesion (injury) in medical practice, while limiting the false positive number that lead to pointless and discomforting biopsies.Computer aided design frameworks in regards to mammography may address two unique undertakings: recognition of suspicious injuries in a mammogram (CADe) and diagnosis of recognized blisters/lesions (CADx), i.e., grouping as malignant or benign. Profound learning is viewed as a huge leap forward innovation of ongoing years as it has shown the best performance in different undertakings of tasks based on machine learningprocess comprising object classification and its detection.Recently, deep learning (DL) strategies, extraordinarily CNNs (likewise called asConvNets) have picked up bunches of considerations to CAD for mammographs as they help in defeating the limitations of CAD frameworks' [2, 41_43, 69]. CNNs accomplish higher discovery exactness than the models of $\mathrm{CAD}$, and benefitthe radiologists progressively providing more precise conclusion/diagnosis by conveying quantitative investigation of suspicious injuries $[4,12]$. An ongoing [48, 72][74] examination study demonstrates that utilizing DL techniques lowers the error rate of humans for the breast cancer diagnoses by $85 \%$. Existingprototypes of $\mathrm{CNN}$ are intended to increase radiologists' capacity to discover even the tiniest malignant growths at their most punctual stages alarming the radiologist to the requirement for further investigation [5, 71][78]. Furthermore, propels in CNNs can help radiologists, yet in addition in the long run make conclusion frameworks to read MGs freely soon [70, 71]. Over the last couple of years, CNNs have prompted achievements in a huge variety of pattern classification and recognition issues for normal graphics because of the accessibility of enormous information storehouses, quick processing of graphical units, and the intensity of distributed and parallel computing[38][71, 73].Training a profound model of CNN [7] with a constrained numberof restorative information is thought-provoking that has been talked by utilizing the tehniques of augmentation and transfer 
learning (TL) [38]. Studies demonstrate that CNN strategies that analyze pictures of breasts from left and right [68]and additionally the mediolateral-angled (MLO) and cranio caudal (CC) perspective on each section of breast can increase the ability of accuracy exposureand diminish the false positives rates [5]. CNNshave additionally been utilized in the applications of risk evaluation to build the precision of recognizing breast malignancy by the radiologist $[64,65]$.

\subsection{Screening in mammography}

The disease of breast cancer is the most well-known malignant growth in ladies and it is the fundamental driver of death from malignancy among ladies around the globe [1]. Mammography Screening has usually appeared to decrease the mortality of breast cancer growth by $38-48 \%$ among members [2, 15]. During a typicalexamination of mammography screening, the X-ray pictures are caught from 2 points of each section of the breast part. These pictures are examined for harmful lesions by a couple of skilled radiologists. For further indicative assessment, doubtful cases are called back. Screening based on mammograms isusually assessed by human readers [34, 36][55]. The procedure of reading is tiring, dull, protracted, exorbitant and in particular, inclined to faulty errors. Various examinations have demonstrated that around $20-30 \%$ of analyzed malignant growths might establish reflectively on the past test based on negative screening test bby means of blinded commentators. The issue of neglected tumors still continues in spite of current FFDM (full field digital mammography). The particularity and sensitivity of broadcasting mammography is accounted to lieamid $89-97 \%$ and $77-87 \%$ individually. Twofold reading was set up to recover the concert of mammographic assessment and it has been executed in numerous nations. Different analyses can additionallyrecover symptomatic execution up to in excess of 10 readers, demonstrating that there is opportunity to get advancement in mammogram assessment further than the process of twofold reading $[70,32][23]$.

\subsection{CAD in mammography screening}

CAD arrangements were created to support radiologists in examining the research based on mammograms. These projects as a rule break down a mammogram and imprint the doubtful areas, which ought to be inspected by the radiologist [5]. The innovation was endorsed by FDA and had widen rapidly. By the year 2008, U.S has reported that in the Medicare populace, $74 \%$ of all screening mammograms were deciphered with CAD, anyway the expense of CAD use is above $\$ 400$ million every year [11]. The advantages of utilizing $\mathrm{CAD}$ are questionable. At first a few investigations have indicated promising outcomes with CAD [6][70]. An enormous clinical preliminary in the UK has demonstrated that solitary perusing with CAD help has comparable execution to twofold perusing or reading [60]. Nonetheless, in the most recent decade various examinations inferred that as of now utilized CAD innovations don't advance the presentation of radiologists in regular practice [11][56]. These dubious outcomes show that CAD frameworks should be improved before radiologists can at last profit by utilizing the innovation in regular practice. Presently, the CAD methodologies [17, 23][44, 45, 49, 58] depend on depicting a $\mathrm{X}$-ray graphic with fastidiously planned machine learning and handmade features for arrangement on top of these taken features. Since 2012, in the area of PC vision, profound CNNs have essentially beated these conventional techniques [28, 59]. Deep or Profound CNNs have outperformed well, human execution in object identification and the classification of image [18]. These kind of models have enormous potential in the examination of medical image. A few investigations have endeavored to apply Deep Learning to examine mammograms [6][7, 30_34] however the issue is still a long way from being understood.

\subsection{Source and Methodology}

1.3.1 Data Source:Mammograms with explanations based on pixel-levelwere expected to prepare a lesion-based detector in order toexamine the localisation and classification-based execution. The model is trained on the open DDSM [35] known to be Digital Database for Screening Mammography and a set of data from Budapest located Semmelweis University, and it was later tried and verifiedon the open publically built INbreast [54_57] dataset. The pictures utilized for the training phase contains either benign lesions or histologically demonstrated tumors which were reviewed for further examinations, however later ended up being non-malignant. The researchers have expected that phase of training with the two sorts of injuries will assist the model with finding more sores of interest, and separate them among benign and malignant instances [66].

1. DDSM dataset: It comprises of 2620 digitized screening tests of mammography, with ground truth pixel-level mark of lesions. The cancer-basded lesions contin histological evidence. The experts have just utilized the database of DDSM for preparing the model of training and not assessing it. The nature of film-screeneddigitized mammograms isn't in the same class as full-field advanced mammograms subsequently assessment on such kind of cases isn't applicable. The analysts have changed over the lossless jpeg-based pictures to png design, the pixel-values were mapped to optical thickness utilizing adjustment capacities from the website of DDSM, and rescaled the values of pixel to the a range lying between $0-255$.

2. Semmelweis University dataset (Radiology Division): It comprises of 847 pictures of FFDM based on 214 tests from around 174 patients, noted with a gadged named as Hologic LORAD Selenia gadget. Organized board endorsement was acquired for the dataset. Such kind of dataset remained not accessible for the full time of the DM based test, in this manner it was utilized for development in stage second of the DM test, after the explanation based on pixel-level by the creators.

3. INbreast dataset:Comprises 115 cases of FFDM with ground truth pixel-level explanations, along with histological verification for cancer disease [54]. The analysts have amended the pixel level INbreast annotations to outfit the situation of testing. The specialists have overlooked every single kindhearted explanation, and changed over the annotations of malignant lesion to jumping boxes. The experts have avoided 8 of the tests which had vague different discoveries, past medical procedures, artefacts, or questionable obsessive result. The pictures had low contrasting feature, consequently the pixel 
level windows were adjusted. The value of pixel were cut to be most extreme 800 pixels higher and least 500 pixel lower than the method of distribution of the pixel-value (barring the foundation) and were re-scaled to a range lying between 0-255.

\section{Accessibility of data source}

- Semmelweis

University

dataset: (http://semmelweis.hu/radiologia/) was mainly utilised as a superior license consequently is not available openly, though the novelists can provide the data based

upon realisticappeal and authorisation from Semmelweis University.

- DDSM dataset: accessible http://marathon.csee.usf.edu/Mammography/Database. html.

- The INBreast dataset: available at http://medicalresearch.inescporto.pt/breastresearch/ind ex.php/Get_INbreast_Database

\subsubsection{Methodology}

1. Faster $R-C N N$ : This methodology basically depends on a CNN with extra parts for distinguishing, confining and arranging objects in a graphic [40, 42]. R-CNN faster form has a part of convolutional layers, known as RPN called as Region Proposal Network, over the last primary system of theconvolutional layer, which is prepared to distinguish and restrict objects on the graphic, paying little respect to the object class. It utilizes default discovery boxes with various aspect ratios and sizes so as to discover objects with fluctuating shapes and sizes. The most noteworthy scoring defaulty boxes are known to be region recommendations for the other part of the system. The additional part of the neural system assesses the signal originating from each of the proposed area of the last CNN layer, re-sampled to a fixed size. The two branches attempt in solving the task of classification in order to identify theobject presence, and a task of box regression so as to improve the object limits existing in the area. From the identified overlapped objects, the finest expectations are chosen utilizing non-maximized suppression. Additionally, insights regarding Faster form of R-CNN can initiate in the base article [42]. A framework of the exemplaryis shown in Figure.1.The CNN base utilized in the model was a VGG16 network, known to be a profound CNN i.e. 16 layered $[28,43]$. The last layer can recognize 2 sorts of objects in the pictures, malignant or benign lesion. The model's yield represents a bounding box for each distinguished score, and a lesion, which mirrors the trust in the lesion class. To depict a picture with a single score, the experts evaluate the limit of the scores of every malignant injury distinguished in the picture. For different pictures of a similar breast, the average scores of individual pictures is taken. This methodology was spurred by a past report on autonomous human perusers (readers), and it has demonstrated sensibly powerful, while being both basic and adaptable [14].

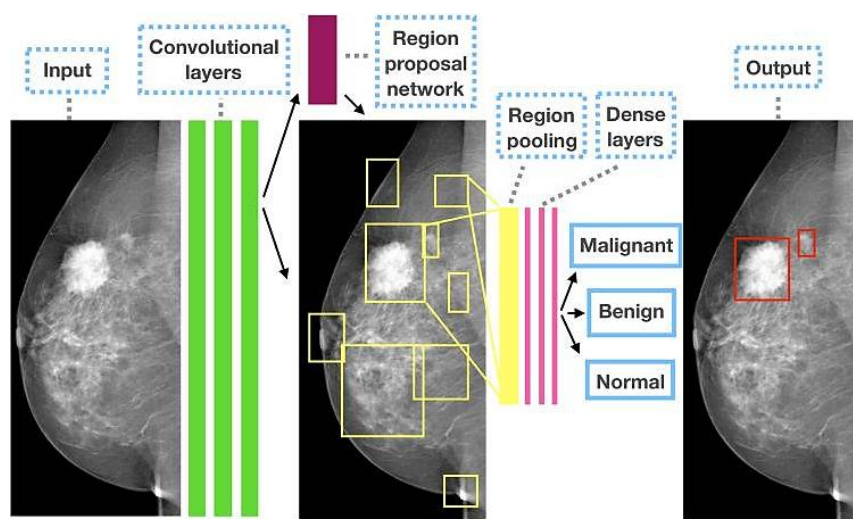

Figure 1: CAD based Faster R-CNN model in mammography

[2]

\subsection{Cancer classification}

1. Model Used:Publically open dataset (INbreast) with the ROC (receiver-operating-characteristics) metric. This dataset covers numerous tests with just a single laterality, hence the experts have assessed expectations for each of the breast section. The framework accomplished AUC i.e. Area Under the ROC Curve $=0.95$, (95 percentile interim: 0.91-0.98, evaluated from bootstrap 10000 testing samples). AUC estimates the whole $2 \mathrm{D}$ region. This is the most elevated AUC score that gave an account of the INbreast dataset with a completely automatic framework dependent on a solitary model, to the best of the analyst's information.

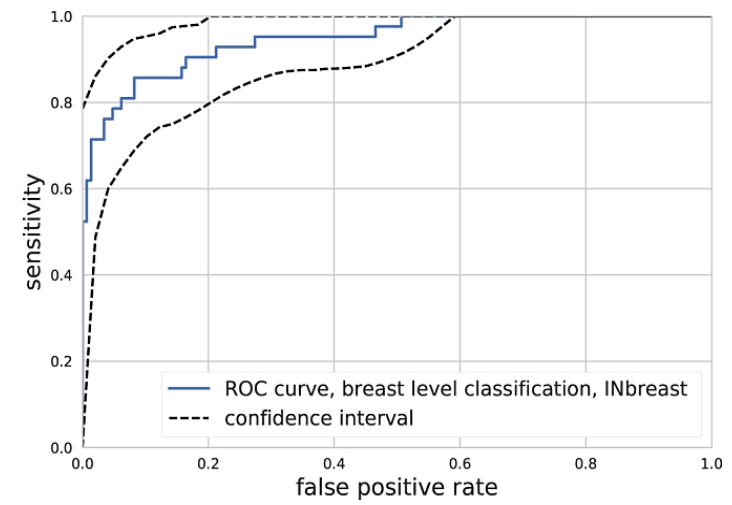

(a)

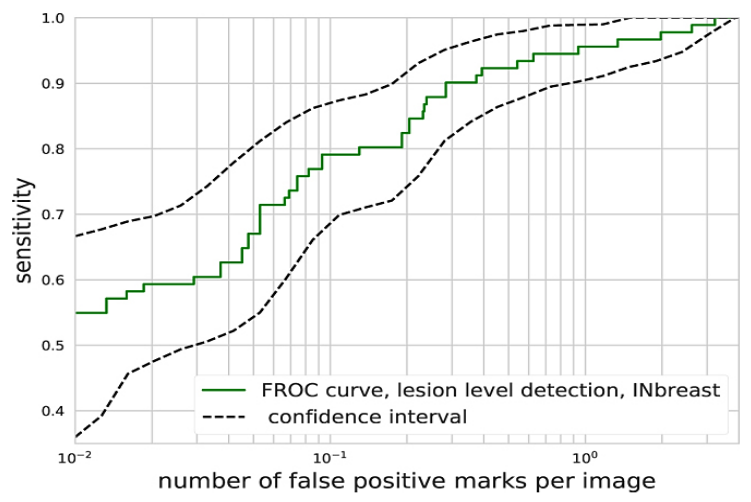

(b)

Figure 2(a): Performance based on classfication. The strong line in blue demonstrates the ROC curve over INbreast based on the level of breat, AUC $=0.95$, the dashed lines demonstrate the 95 percentile interim of the curver/bend dependent on 10000 bootstrap testing samples. (b) FROC bend on INbreast based dataset. The amount of sensitivity is usually evaluated on the basis of 
per lesion methodology. The strong bend with squares demonstrates the outcomes utilizing all pictures, while the dashed lines demonstrate the 95 percentile interim from 10000 bootstrap testing samples [2]

2. FROC Analysis: So as to examine the capacity of the model in order to identify and precisely limit threatening(malignant) sores, the expectations on the INbreast-basedd dataset utilizing the FROC i.e. Free-reaction ROC bend have been accessed in [47]. The FROC based curve demonstrates the sensitivity (division of effectively confined sores) as an element of the quantity of false positive imprints put on a picture Figure 2 (b). As found in Figure 2 (b), [11, 16_20, 59_63] the model had the option to identify malignant sores with 0.3 false positive and 0.9 sensitivity imprints per picture. The announced false positive imprints number per picture for monetarily accessible CAD frameworks ranges from 0.3 to 1.25. The lesion-based sensitivities of economically accessible CAD frameworks are commonly answered to lie in the range $0.75-0.77$ for examined film-based mammograms [19][21_26,46], and 0.85 examined for the case of FFDM $[52,61]$. The model accomplishes marginally better discovery execution on the dataset of INbreast than the announced qualities of CAD frameworks, despite the fact, it is really essential to remind that outcomes acquired on various datasets are not legitimately equivalent. To exhibit the errors and the attributes of the detector, an accumulation of effectively arranged, missed harmful lesions and false positive of the used dataset is done as shown in Figure 3. The score-based threshold for the models was characterized at 0.3 false positive and 0.9 sensitivity imprintsper picture. Subsequent to assessing the location of false positives, the experts have found that most were favorable calcifications or masses. A portion of the benign injuries were biopsy verified by the case depictions of the INbreast. Whereas around $10 \%$ of the dangerous ground truth sores were neglected at the threshold identification, but these values were not totally neglected by the model. With a notch limit which is compared to three kinds of false positive imprints for each picture, every one of the sores were effectively distinguished, see Figure 2(a). Note, that the definite quantity of false-positives and genuine positive recognitions somewhat fluctuates with various instances of pictures, shown by the region as depicted in Figure 2(a).
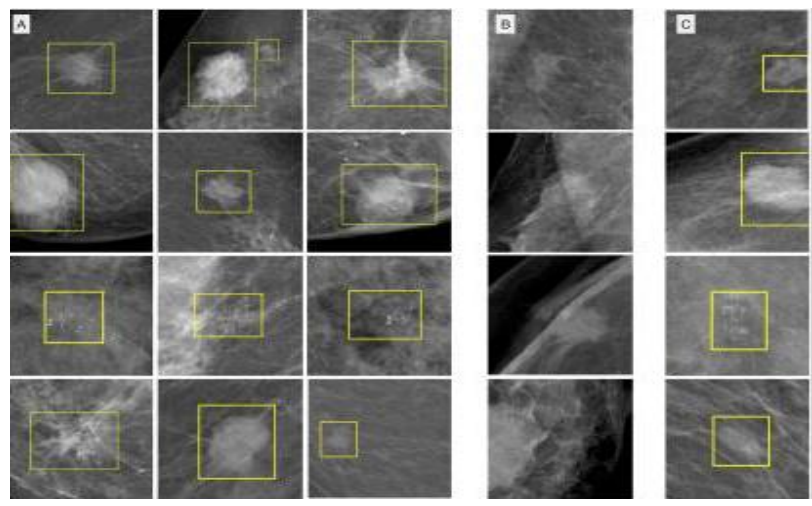

Figure 3: Instances of Detection: The yellow-lined boxes demonstrate the sore in the model. The edge (threshold) for these recognitions was chosen to be at the 0.9 sensitivity detection of lesion. (A) Acceptably distinguished malignant injuries. (B) Neglected malignant injuries. (C) False-positives locations [data obtained form Breast Research Group, INESC Porto, Portugal[2].

\section{RELATED WORK}

\subsection{Principles for inclusion/exclusion of studies in the review}

A far reaching literature-based research, usingthe characterized keywords has been presented in Table 1, on the scientific proceedings and on journals, yet these arenot restricted to the accompanying logical databases: ACMDigital Library, Scopus, IEEE Explore, Science Direct, Web of Science, PubMed. Altogether, 83 of the studies were considered from the timespan of 1995 to November 2017. These examinations center around executing CNNs forlesion confinement and discovery, imageretrieval, risk assessment, high goals picture recreation and the tasks of classification in MG pictures. The criteria of inclusion/exclusion is utilized for this audit are exhibited in Table 1.

Table1: Exclusion/Inclusion for a systematic type of review

\begin{tabular}{l|l} 
Category & Criteria \\
\hline Databases & Public and Private \\
Time period & Available from 1995 to current (Nov 2017). \\
\hline Research focus & $\begin{array}{l}\text { CNN Implementation for the study of breast cancer in } \\
\text { Mammography }\end{array}$ \\
\hline $\begin{array}{l}\text { Publication } \\
\text { Abnormalities }\end{array}$ & $\begin{array}{l}\text { English printed articles } \\
\text { Calcification, Architectural distortion, A symmetries, } \\
\text { and Mass }\end{array}$ \\
\hline Keywords & $\begin{array}{l}\text { Convolutional neural networks, } \\
\text { Mammography, Transfer learning Deep learning, and } \\
\text { Breast Cancer }\end{array}$ \\
\hline
\end{tabular}

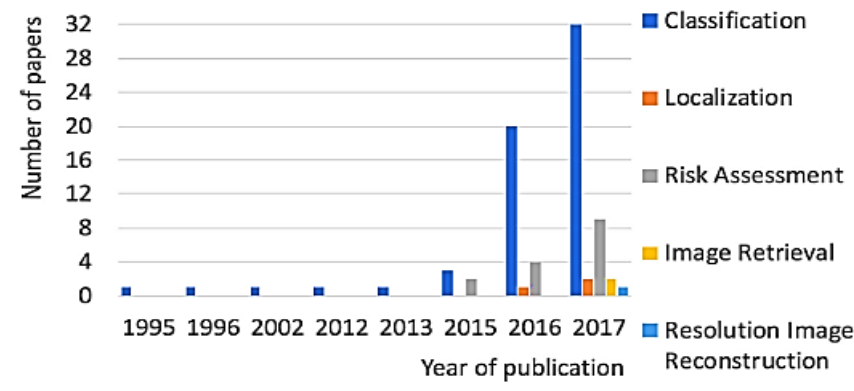

Figure 4 demonstrates a breakdown of the examinations incorporated into this review in the time of production gathered by their NN task.

In this study,we tended to the accompanying exploration: CNN for identifying variations from the norm in MGs, undertakings of the executed $\mathrm{CNN}$, databases, database measure, picture goals, picture type, irregularities associated with the advancement of the CNN, procedures utilized for the arrangement and dataset pre-processing of the informational collection, execution of profound systems on medicinal pictures explicitly MGs, superlative practices that were connected to expand the exactness of discovery of anomalies, regular toolboxes utilized in mammography, and so forth.

\subsection{Digital repositories of Breast cancer}

The databases of Mammography perform a significant job in the phases of testing,training, and assessment of DL techniques. The quantityof information expected to train or prepare a DL system is huge as comparedto the information expected to prepare CNNs.The accessibility of wide-spread noted databases isprecarious for propelling DL advancement in the field of medical imaging. The most regular discoveries perceived on mammographyare irregular mass area, architectural distortion (AD), asymmetries, and calcifications. In common there are various freely accessible databases for Mammography (MG): DDSM [19], INbreast database [50], MIAS (Mammographic Image

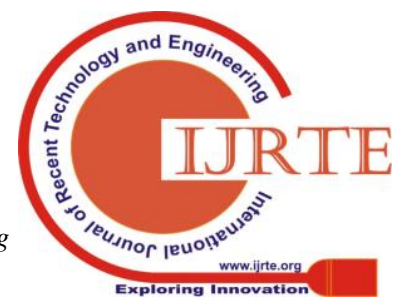


Analysis Society) [36], BCDR (Breast Cancer Digital Repository) [52, 53], IRMA (Image Retrieval in Medical Applications) [61]. Table 2 basically conpares accessible MG databases that area available publically based on the primary initial beginning, the quantity of pictures, images size, views of MLO and CC, film or computerized database, thepictures formar, pictures goals, and the process of disseminationof malignant, benign, and normal images. Various kind of databases utilized in the part of literature are private in nature and are confined toindividual associations [8, 26, 27, 29, 9, 49-52]. Thedatabases (public) presents a large unevenness ofpatient's and a blend of malignant, benign, and normal cases. The open (publically built) depositorieshave gathered a film screen mammographs (MGs) known as FSMs [21, 36, 38 , 39], or computerized digital mammography [38, 39, 47] with various goals.

(a) INbreast database: It is increasing more consideration these days and utilized in $[12,13,40,57]$. Its focal points have high goals and precise division of injuries; its little size and the constrained shape mass varieties are its downsides.

(b) MIAS database:MIAS [49-51] database pictures have solid noise and low resolution. It represents an old database system that contain images limited in number. In spite of every one of these disadvantages, it has been generally utilized in section of literature till today.

(c) IRMA project: It is a mixture of various databases based on different sizes and goals. However, ROI (Region of Interest) explanations for such kind of databases are increasingly precise in nature making them progressively precise for DL techniques.

(d) DDSM database:The images of DDSM [9, 10, 11, 32, 14] are usually saved in a non-standard compressed document that need utilization of decompressing codes. Besides, the annotations of ROI for the variations in the images of DDSM demonstrate general location of injuries/lesions, without exact division of them.

(e) BCDR: It presents anauspicious database yet it requires the improvement stage. The methodology of BCDR has been utilized in various kind of examinations $[12,32,52]$. The qualities and impediments of these databases are abridged in Table 3.

Table 2: Comparative analysis between broadly utilized databases in the literature section w.r.t size of pictures, bits/pixel (bpp), views (MLO, CC) film or digital databases, and the typical distribution of malignant, benign, and normal pictures. [CC known as Cranial-Caudal, MLO known as mediolateral-diagonal, angled or an obliqueview)

\begin{tabular}{|c|c|c|c|c|c|c|c|}
\hline Database & $\begin{array}{c}\text { Size of } \\
\text { Image }\end{array}$ & Views & Type & Bpp & *Normal & *Benign & $\begin{array}{c}* \text { Malignan } \\
\text { t }\end{array}$ \\
\hline MIAS & $1024 \times 1024$ & MLO & FSM & 8 & 207 & 69 & 56 \\
\hline INbreast & Several & Both & FFDM & 16 & 67 & 220 & 49 \\
\hline DDSM & $3118 \times 5001$ & Both & FSM & 12 & 914 & 870 & 695 \\
\hline IRMA & Several & Both & Both & 12 & 1108 & 1284 & 1284 \\
\hline BCDR-F01 & $720 \times 1168$ & Both & FSM & 8 & 0 & 187 & 175 \\
\hline BCDR-F02 & $720 \times 1168$ & Both & FSM & 8 & 0 & 426 & 90 \\
\hline
\end{tabular}

Table 3:An instant summary for the limitations and strengths of the MIAS, INbreast, BCDR, DDSM, and IRMA databases

\begin{tabular}{|c|c|c|}
\hline Database & Limitation & Strength \\
\hline MIAS & Limited size & Still widely used, Different resolutions. \\
\hline INbreast & $\begin{array}{l}\text { Limited size, Limited mass } \\
\text { shapevariations, Old database, }\end{array}$ & $\begin{array}{l}\text { Standard file format, Accurate position of } \\
\text { lesions }\end{array}$ \\
\hline DDSM & Not precise, Non-standard format & $\begin{array}{l}\text { Widely used database, Different lesions shape } \\
\text { variations. }\end{array}$ \\
\hline IRMA & Non-standard format & Accurate position of lesions, High resolution \\
\hline BCDR & Limited size. & $\begin{array}{l}\text { Standard file format, Accurate position of } \\
\text { lesions, Still in thr phase of development. }\end{array}$ \\
\hline
\end{tabular}

\subsection{Convolutional neural network}

In 1995, CNNs were utilized to examine breast malignancy $[41,76]$. Renowned CNNs, for example, ZF-Net[43], Alex-Net [51], VGG-Net [45], ResNet [28], and GoogLeNet [39] have realized leaps forward in processing images.The design of Alex-Net is widely utilized in the medical-imaging area for the detection of breast malignancy. Since the year 2012, CNNs have turned out to be progressively mainstream and have pulled in more consideration as a result of the expanding the power of computing, accessibility of lower cost equipment, the ascent ofbig information, and the open source of algorithms. CNNs[8, 77] structure is fundamentally the same as that of common neural systems. The essential CNN design is a heap of kind of pooling layer (Max-pooling), convolutional layer, nonlinear layer (ReLU), and a loss function(for instance Softmax/ SVM) on the last completely associated fully connected (FCs) as depicted in Figure. 4. 


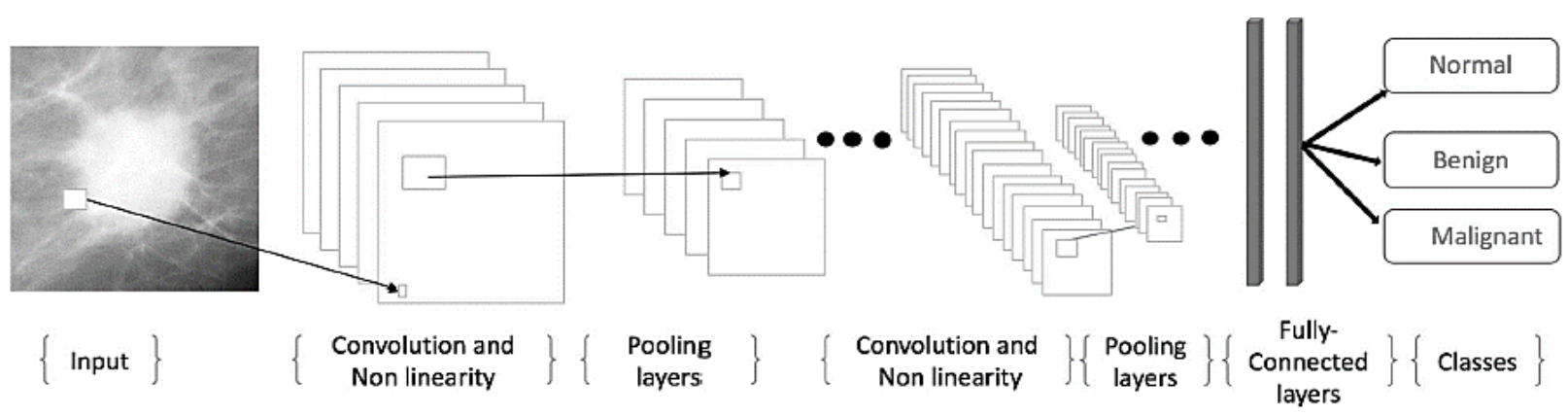

Figure 4: CNN architecture[1]

The yield can be a solitary class (for example malignant, benign, normal) or a likelihood of classes that best depicts the picture. The input-based contribution to a convolutionlayer isan picture $(\mathrm{W} 1 \times \mathrm{H} 1 \times \mathrm{D} 1)$. Here $\mathrm{W} 1$ represents the width, $\mathrm{H} 1$ represents tallness of the picture and D1 represents the quantity of channels, for instance a RGB picture contains $D 1=3$. The convolutional layer contains $F$ filters (for example 12 kind of filters) having $\mathrm{N} \times \mathrm{N} \times \mathrm{D} 1$ size where Nis slightly smaller than the image dimension and D1 is equivalent to the quantity of channels (for instance $5 \times 5 \times 3$. Here, 5 pixels is the height and the width, and 3 since pictures have profundity (depthness) 3. .During the operational activity of convolution, each one of the filter $\mathrm{F}$ gets convolved with the picture to deliver K-basedmapping features of volume size
$(\mathrm{W} 2 \times \mathrm{H} 2 \times \mathrm{D} 2)$ estimate where: $\mathrm{W} 2=\mathrm{H} 2=(\mathrm{W} 1-\mathrm{F}+2 \mathrm{P}) / \mathrm{S}+1$. Here $\mathrm{S}$ is the quantity of steps, $\mathrm{P}$ presents the measure of mecahnsism based on zero-padding, and D2=F. For each component of the feature map, anactivation function based on non-linear nature is applicable (for instanmce ReLU). The non-linearisedactivation functionleaves the volume size unaltered $(\mathrm{W} 2 \times \mathrm{H} 2 \times \mathrm{D} 2)$. In the wake of employing ReLU, a down-testing activity called Pool is connected beside the spatial measurements (height, width) of the outcome-based feature map. In the wake of pooling layer, there might be any number of completely associated layers that calculate the scores class as shown in Figure. 4.

Table 4: The structures of ZF-NET, AlexNET, VGG-NET, and GoogLeNET

\begin{tabular}{|c|c|c|c|c|}
\hline Popular CNNs $\square$ & ZF-NET & AlexNET & VGG-NET & GoogLeNET \\
\hline Year & 2013 & 2012 & 2014 & 2014 \\
\hline Number of layers & 8 & 8 & 19 & 22 \\
\hline Image Resolution & $227 \times 227$ & $227 \times 227$ & $224 \times 224$ & $224 \times 224$ \\
\hline $\begin{array}{l}\text { Number of } \\
\text { Conv-Pool layers }\end{array}$ & 5 & 5 & 16 & 21 \\
\hline $\begin{array}{l}\text { Number of FC } \\
\text { layers }\end{array}$ & 3 & 3 & 3 & 1 \\
\hline $\begin{array}{l}\text { Full connected } \\
\text { layer size }\end{array}$ & $4096,4096,1000$ & $4096,4096,1000$ & $4096,4096,1000$ & 1000 \\
\hline Number of Filters & $96-384$ & $96-384$ & $64-512$ & $64-384$ \\
\hline Filter Sizes & $3,5,11$ & $3,5,11$ & 3 & \\
\hline Dropout & + & + & + & + \\
\hline $\begin{array}{l}\text { Data } \\
\text { Augmentation }\end{array}$ & + & + & + & + \\
\hline Number of GPU & 1 GTX & 2 GTX & 4 Nvidia & A few high-end \\
\hline Training Time & 12 days & 5:6 days & $2: 3$ weeks & 1 week \\
\hline Top error & $11.2 \%$ & $16.40 \%$ & $7.30 \%$ & $6.70 \%$ \\
\hline
\end{tabular}

\subsection{Mamogram Analysis}

The upgradation process of mammogram and the algorithms of segmentation are intended for mammograms taken from diverse kind of views that involve: $\mathrm{CC}$ i.e. cranio-caudal and MLO i.e. medio-lateral oblique view. The algorithm of mammogram investigation comprises of five phases depicted in the following section:

Stage 1: It is known as the primary phase of the proposed algorithm as represented in Figure. 1, the segmentation of breast part is connected and it involves three type of stages: in the primary stage, labels, borders, and supplementary artifacts are expelled from the image of mammogram. In stage second, the noise is expelled so as to improve the mammogrphic pictures. At last, the thirdstage involves theprocess of breast region segmentation utilizing contour-based segmentation.

Stage 2: In request to explainthe masses associated with breast, the segmented section of the breastarea is upgraded in this phase utilizing two improvement approaches: un-sharp masking and histogram balance. The later one represents a method of contrast enhancement; it alters the intensities of pixel inorder to get another improved picture with generally expanded form of local contrast. The former mentioned, is the way toward sharp pictures by 
subtraction of a smoothed form of a picture from its unique. The method of histogramequalization can be connected selectedregions or the whole picture. The first histogram is made for the picture, utilizing theprobability of event of pixels power/intensities given as follows [40, 67]:

$$
P_{r}\left(r_{k}\right)=\frac{n_{k}}{M N}
$$

Here, $P_{r}\left(r_{k}\right)=$ likelihood occurrence of $r_{k}$ intensity level, $\mathrm{MN}=$ absolute number of pixels, $n_{k}$ represents the the quantity of pixels that having intensity $r_{k}$. The evaluation of new intensity values for every level of intensity is finished utilizing thefollowing transformation or change (mapping):

$s_{k}=T\left(r_{k}\right)=(L-1) \sum_{j=0}^{k} P_{r}\left(r_{k}\right)$

$s_{k}=$ new level of intensity, and is intended for each of the pixel in the image.

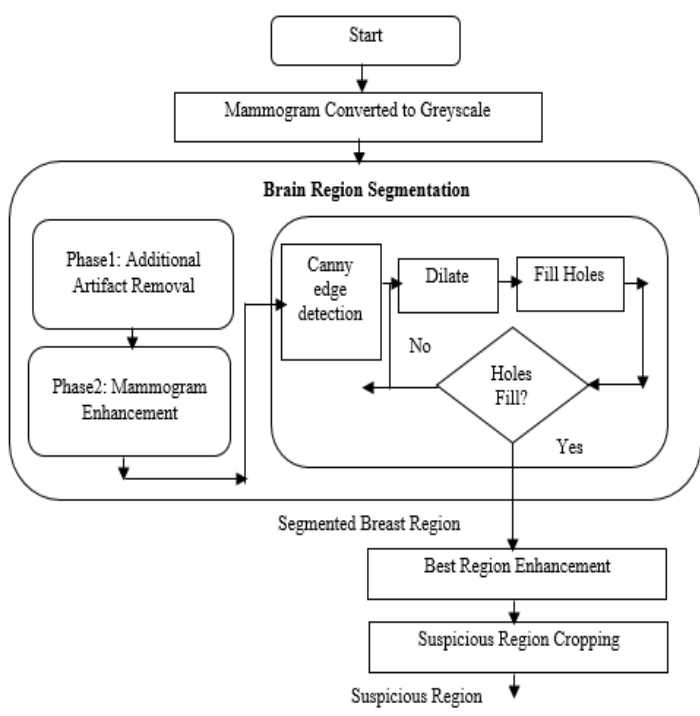

Figure 5: Breast region segmentation.

This research study involves thefollowing improvementtechniquesthat were usually applied by the radiologist and further verified by the the criteria based on Good Segmentation.

1. CLAHE: Namely, Contrast Limited Adaptive Histogram Equalization algorithmworks on little areas of the gray image by segregating the picture into relevant areas named tiles. Then the method of histogram equalization is applied to each of the tile's contrast, accordingly, the gray values distribution is evened, consequently the concealed image features turn tobecome progressively observable.

2. Median filtering:In the class of the order-static filters, it is usually considered as one of the best filter. In the presence of impulse noiseMedian filters are likewise viable. It canbe connected on the mammograms before division/segmentation so as to lessen the measure of preserved edges and noise.

3. Gaussian smoothing:Gaussian filter (low pass) is mainly utilized tosmooth the images of mammogram. For the most part, the frequency-based filtering depends on the FT. In this manner, the administrator takes a filter function nd the image in the Fourier space/domain. At that point the image gets multiplied on pixel-by-pixel basis with the help of a filter function as the following [62]:

$G(u, v)=F(u, v) \times H(u, v)$ where $H(u, v)=e^{-D^{2}(u, v) / 2 r^{2}}$
Here, $H(u, v)=$ filter function, $G(u, v)=$ resulted filtered image, $D(u, v)=$ frequency rectangle based distance from the center andrepresents the cutoff frequency.

A low-pass filtering channel lessens large frequencies and holds low frequencies unaltered. The outcome inspatial area is proportional to that of a smoothing channel, as thehigh blockingfrequencies relate to high-pitched changes in intensity.

Stage 3: In this stage, the doubtfulpart is trimmed from theimproved region of breast, and the first mammogram picture is also trimmed utilizing similar measurements in an automatic manner. The image cropped is then utilized as contribution for stage 4, as appeared in Figure 5.

Stage 4: This stage comprises of four type of stages. In the first stage, the image is improved utilizing the acquired un-sharp mask. Secondly, the image gets changed over into a twofold (binary) picture by means of a system known as Otsu thresholding system [67]. The outcome is expelled from thetwofold picture utilizing morphological administrators. Thirdly,the objects associated on fringes are expelled from the binary picture created. At last, openingsof specific sizes are detached utilizing morphological administrators [62].The technique based on Ostu thresholding gets utilized in stage 2 . Otsu thresholding technique: Utilized in stage 2 scans thoroughly for the edge or threshold that limits the cariance based on intra-class mechanism, well-defined as variance-based weighted sum of two of the classes: $\sigma_{w}^{2}(t)=w_{1}(t) \sigma_{1}^{2}(t)+w_{2}(t) \sigma_{2}^{2}(t)$, where, $w_{i}$ weights represents the probabilities of both classes isolated by threshold $(\mathrm{t})$ and $\sigma_{i}^{2}$ the class variances.

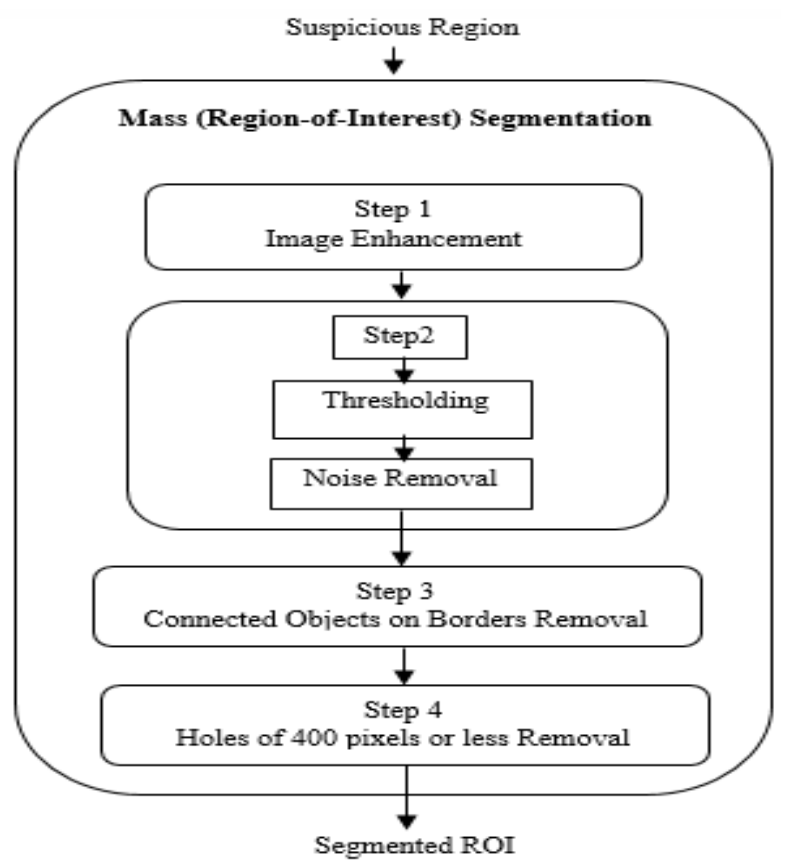

Figure 5:Mass Segmentation

Stage 5: As appeared in Figure 5, this stage involves the preceding of the mass segmented, the limit of the ROI is outlined utilizing the limit (boundary) extraction morphologically built algorithm. 
Gap (Hole) filling: A gap can be characterized as a foundation an area enclosed by a border associated with closer view pixels. The main objective of the algorithm involves the filling of the gaps for having binary pictures. The algorithm of hole-fillingrequires two sources of input, set comprising gaps, and a component of symmetric-based structuring, andis characterized as: $\mathrm{Xk}=(\mathrm{Xk}-1 \oplus \mathrm{B}) \cap \mathrm{A}^{\mathrm{C}}$ for $\mathrm{k}=1,2,3, \ldots, \mathrm{n}$. Here $\mathrm{B}$ represents the symmetric-based organizing component, and the algorithm ends at the step of iteration $\mathrm{k}$ if $\mathrm{Xk}=\mathrm{Xk}-1$. Xk as the set thereby contains all the filled gaps; the association of $\mathrm{A}$ and Xkencompasses all the hoes filled along with their limits. The crossing point at each progression with a type A complement bounds the outcome to ROI.

Boundary (Limit) extraction: The limit can be gotten by applying the process of eroding, and further it involves the substraction of the image eroded from its unique form, and is characterized as: $\beta(A)=A-(A \Theta B)$, here $\beta(A)$ is known as the resulting boundary, $A$ depicts realtype of image, and $B$ is

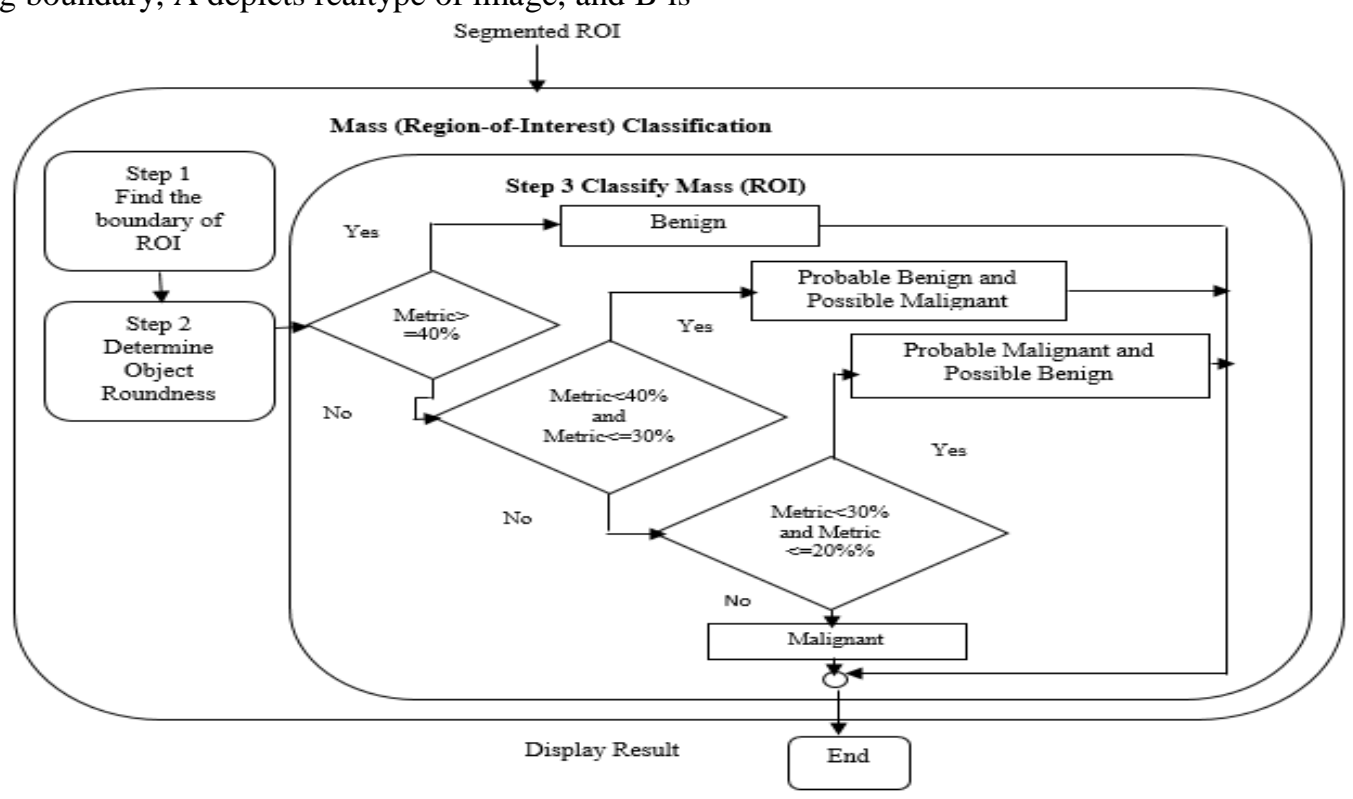

Figure 6:Mass Classification known as the component of structuring or an element. Each of the mass can be arranged into one of the accompanying four kind of classes: probable benign, possible malignant, benign, possible benign, or malignant. This kind of arrangement is generally confers to the accompanying criteria (in view of radiologists' proposal):

1. Deciding the object or item roundness: In this progression, the perimeter and the area is evaluated for the mass, at that point these outcomes are used to establish the mass roundness utilizing the accompanying condition:

$$
\text { metric }=4 \times \frac{\pi \times \text { area }}{\text { parameter }}
$$

In the event if the metric of the system equals to one, at that point the mass is circle, else it is of different shapes.
2. Categorize into malignant or benign in response to a particular threshold system: This edge or threshold is usually determined by joining the outcomes of metric-based condition got from the past step for both CC and MLO mammogram pictures depending upon the following condition:

$$
\text { Average metric }=\frac{M L O_{\text {metric }}+C C_{\text {metric }}}{2}
$$

\section{CONCLUSION}

Breast disease or cancer is one of the real reasons for death among ladies. Because of the wide scope of highlights related to breast variations, a few irregularities might be confused or missed. There is additionally various false positive discoveries and in this way a great deal of superfluous biopsies. The algorithms base on CAD have been created to enable radiologists to give a precise finding and to lessen the quantity of false positives. In this investigation, distinctive steps in the algorithms of have been widely examined. The methods in the field of CAD based mammography incorporates pre-processing of image, procedures of image segmentation, feature selection, feature extraction, mammograms features, and the techniques if classification. Further advancements in every step of algorithm is essential to recover the general execution of CAD supported detection and diganosis. In the study of image segmentation, the outline of different strategies of based on digital image processing is concisely described. The strategy of textural analysis is presented to classify malignant and benign masses and to recognize the calcification in the process of mammography.The techniques of segmentation and mass detection, when all is said in done, are still needing improvement. In this sense, mammographic algorithms based on mass segmentation (division) or detection (location)utilizing a solitary picture are relied upon to improve in various ways. Genuine patterns in the acknowledgment of unsupervised object depends uponvisual vocabularies and probabilistic inactive semantic examination. Despite the fact that these methodologies are outflanking the conventional schemes of unsupervised nature for explicit applications, we accept that managed methodologies are probably going to accomplish a 
superior execution for mass segmentation/detection accordingly methodologies are fit for incorporating the enormous variety in the morphology and size of these variations from the irregularities. Possibly effective segmentation/detection strategies should join both base up data of the question image and top-down compelling data of the article to be recognized.

\section{REFERENCES}

1. Abdelhafiz, Dina, Clifford Yang, Reda Ammar, and Sheida Nabavi. "Deep convolutional neural networks for mammography: advances, challenges and applications." BMC bioinformatics 20, no. 11 (2019): 281.

2. Ribli, Dezső, Anna Horváth, Zsuzsa Unger, Péter Pollner, and István Csabai. "Detecting and classifying lesions in mammograms with deep learning." Scientific reports 8, no. 1 (2018): 4165.

3. Litjens G, Kooi T, Bejnordi BE, Setio AAA, Ciompi F, Ghafoorian M, et al."A survey on deep learning in medical image analysis". 2017. arXivpreprint arXiv: 170205747

4. Lee JG, Jun S, Cho YW, Lee H, Kim GB, Seo JB, et al. Deep Learning in Medical Imaging: General Overview. Korean J Radiol. 2017;4 (18):570-84

5. Wang, Juan, Huanjun Ding, Fatemeh Azamian Bidgoli, Brian Zhou, Carlos Iribarren, Sabee Molloi, and Pierre Baldi. "Detecting cardiovascular disease from mammograms with deep learning." IEEE transactions on medical imaging 36, no. 5 (2017): 1172-1181.

6. Dhungel, Neeraj, Gustavo Carneiro, and Andrew P. Bradley. "Fully automated classification of mammograms using deep residual neural networks." In 2017 IEEE 14th International Symposium on Biomedical Imaging (ISBI 2017), pp. 310-314. IEEE, 2017.

7. Lotter, William, Greg Sorensen, and David Cox. "A multi-scale CNN and curriculum learning strategy for mammogram classification." In Deep Learning in Medical Image Analysis and Multimodal Learning for Clinical Decision Support, pp. 169-177. Springer, Cham, 2017.

8. Kooi, Thijs, Bram van Ginneken, Nico Karssemeijer, and Ard den Heeten. "Discriminating solitary cysts from soft tissue lesions in mammography using a pretrained deep convolutional neural network." Medical physics 44, no. 3 (2017): 1017-1027.

9. Ahn, Chul Kyun, Changyong Heo, Heongmin Jin, and Jong Hyo Kim. "A novel deep learning-based approach to high accuracy breast density estimation in digital mammography." In Medical Imaging 2017: Computer-Aided Diagnosis, vol. 10134, p. 101342O. International Society for Optics and Photonics, 2017.

10. Yi, Darvin, Rebecca Lynn Sawyer, David Cohn III, Jared Dunnmon, Carson Lam, Xuerong Xiao, and Daniel Rubin. "Optimizing and visualizing deep learning for benign/malignant classification in breast tumors." arXiv preprint arXiv:1705.06362 (2017).

11. Ben-Ari, Rami, Ayelet Akselrod-Ballin, Leonid Karlinsky, and Sharbell Hashoul. "Domain specific convolutional neural nets for detection of architectural distortion in mammograms." In 2017 IEEE 14th International Symposium on Biomedical Imaging (ISBI 2017), pp. 552-556. IEEE, 2017.

12. Jiang, Fan, Hui Liu, Shaode Yu, and Yaoqin Xie. "Breast mass lesion classification in mammograms by transfer learning." In Proceedings of the 5th international conference on bioinformatics and computational biology, pp. 59-62. ACM, 2017.

13. Dhungel, Neeraj, Gustavo Carneiro, and Andrew P. Bradley. "Fully automated classification of mammograms using deep residual neural networks." In 2017 IEEE 14th International Symposium on Biomedical Imaging (ISBI 2017), pp. 310-314. IEEE, 2017.

14. Abbas, Qaisar. "DeepCAD: A computer-aided diagnosis system for mammographic masses using deep invariant features." Computers 5, no. 4 (2016): 28

15. Mammography [Online]. Available https://medlineplus.gov/mammography.html[Accessed 01-07-2019]

16. Kooi, Thijs, Albert Gubern-Merida, Jan-Jurre Mordang, Ritse Mann, Ruud Pijnappel, Klaas Schuur, Ard den Heeten, and Nico Karssemeijer. "A comparison between a deep convolutional neural network and radiologists for classifying regions of interest in mammography." In International Workshop on Breast Imaging, pp. 51-56. Springer, Cham, 2016.

17. Greenspan, Hayit, Bram Van Ginneken, and Ronald M. Summers. "Guest editorial deep learning in medical imaging: Overview and future promise of an exciting new technique." IEEE Transactions on
Medical Imaging 35, no. 5 (2016): 1153-1159. He, K., Zhang, X., Ren, S. \& Sun, J. Delving deep into rectifiers: Surpassing human-level performance on imagenet classification. In Proceedings of the IEEE international conference on computer vision, 1026-1034 (2015).

18. Li, Y., H. Chen, L. Cao, and J. Ma. "A survey of computer-aided detection of breast cancer with mammography." J Health Med Inf 4 , no. 7 (2016)

19. Kooi, Thijs, Bram van Ginneken, Nico Karssemeijer, and Ard den Heeten. "Discriminating solitary cysts from soft tissue lesions in mammography using a pretrained deep convolutional neural network." Medical physics 44, no. 3 (2017): 1017-1027.

20. Ahn, Chul Kyun, Changyong Heo, Heongmin Jin, and Jong Hyo Kim. "A novel deep learning-based approach to high accuracy breast density estimation in digital mammography." In Medical Imaging 2017: Computer-Aided Diagnosis, vol. 10134, p. 101342O. International Society for Optics and Photonics, 2017

21. Yi, Darvin, Rebecca Lynn Sawyer, David Cohn III, Jared Dunnmon, Carson Lam, Xuerong Xiao, and Daniel Rubin. "Optimizing and visualizing deep learning for benign/malignant classification in breast tumors." arXiv preprint arXiv:1705.06362 (2017).

22. Ben-Ari, Rami, Ayelet Akselrod-Ballin, Leonid Karlinsky, and Sharbell Hashoul. "Domain specific convolutional neural nets for detection of architectural distortion in mammograms." In 2017 IEEE 14th International Symposium on Biomedical Imaging (ISBI 2017), pp. 552-556. IEEE, 2017.

23. Jiang, Fan, Hui Liu, Shaode Yu, and Yaoqin Xie. "Breast mass lesion classification in mammograms by transfer learning." In Proceedings of the 5th international conference on bioinformatics and computational biology, pp. 59-62. ACM, 2017

24. Dhungel, Neeraj, Gustavo Carneiro, and Andrew P. Bradley. "Fully automated classification of mammograms using deep residual neural networks." In 2017 IEEE 14th International Symposium on Biomedical Imaging (ISBI 2017), pp. 310-314. IEEE, 2017.

25. Abbas, Qaisar. "DeepCAD: A computer-aided diagnosis system for mammographic masses using deep invariant features." Computers 5 , no. 4 (2016): 28

26. Kallenberg, Michiel, Kersten Petersen, Mads Nielsen, Andrew Y. Ng, Pengfei Diao, Christian Igel, Celine M. Vachon et al. "Unsupervised deep learning applied to breast density segmentation and mammographic risk scoring." IEEE transactions on medical imaging 35, no. 5 (2016): 1322-1331

27. He, Kaiming, Xiangyu Zhang, Shaoqing Ren, and Jian Sun. "Deep residual learning for image recognition." In Proceedings of the IEEE conference on computer vision and pattern recognition, pp. 770-778 2016.

28. Sun, Wenqing, Tzu-Liang Bill Tseng, Bin Zheng, and Wei Qian. "A preliminary study on breast cancer risk analysis using deep neural network." In International Workshop on Breast Imaging, pp. 385-391 Springer, Cham, 2016.

29. Gallego-Posada, J., D. A. Montoya-Zapata, and O. L. Quintero-Montoya. "Detection and diagnosis of breast tumors using deep convolutional neural networks." Medical Physics43 (2016): 3705-3705.

30. Zhu, Wentao, Xiang Xiang, Trac D. Tran, and Xiaohui Xie "Adversarial deep structural networks for mammographic mass segmentation." arXiv preprint arXiv:1612.05970 (2016).

31. Arevalo, John, Fabio A. González, Raúl Ramos-Pollán, Jose L. Oliveira, and Miguel Angel Guevara Lopez. "Representation learning for mammography mass lesion classification with convolutional neural networks." Computer methods and programs in biomedicine 127 (2016): 248-257

32. Siegel, R.L.; Miller, K.D.; Jemal, A. Cancer statistics, 2015. CA Cancer J. Clin. 2015, 65, 5-29.

33. Lehman, Constance D., Robert D. Wellman, Diana SM Buist, Karla Kerlikowske, Anna NA Tosteson, and Diana L. Miglioretti. "Diagnostic accuracy of digital screening mammography with and without computer-aided detection." JAMA internal medicine 175, no. 11 (2015): 1828-1837.

34. Faster, R. "Towards Real-Time Object Detection with Region Proposal Networks Shaoqing Ren [J]." Kaiming He, Ross Girshick, and Jian Sun.

35. Carneiro, Gustavo, Jacinto Nascimento, and Andrew P. Bradley "Unregistered multiview mammogram analysis with pre-trained deep learning models." In International Conference on Medical Image Computing and Computer-Assisted Intervention, pp. 652-660. Springer, Cham, 2015 
36. American Cancer Society. Breast Cancer Facts \& Figures; American Cancer Society, Inc.: Atlanta, GA, USA, 2015.

37. LeCun Y, Bengio Y, Hinton G. Deep learning. Nature. 2015;521 (7553):436-44.

38. Fonseca, Pablo, Julio Mendoza, Jacques Wainer, Jose Ferrer, Joseph Pinto, Jorge Guerrero, and Benjamin Castaneda. "Automatic breast density classification using a convolutional neural network architecture search procedure." In Medical Imaging 2015: Computer-Aided Diagnosis, vol. 9414, p. 941428. International Society for Optics and Photonics, 2015.

39. Carneiro, Gustavo, Jacinto Nascimento, and Andrew P. Bradley. "Unregistered multiview mammogram analysis with pre-trained deep learning models." In International Conference on Medical Image Computing and Computer-Assisted Intervention, pp. 652-660. Springer, Cham, 2015.

40. Szegedy, Christian, Wei Liu, Yangqing Jia, Pierre Sermanet, Scott Reed, Dragomir Anguelov, Dumitru Erhan, Vincent Vanhoucke, and Andrew Rabinovich. "Going deeper with convolutions." In Proceedings of the IEEE conference on computer vision and pattern recognition, pp. 1-9. 2015.

41. Faster, R. "Towards Real-Time Object Detection with Region Proposal Networks Shaoqing Ren [J]." Kaiming He, Ross Girshick, and Jian Sun.

42. Simonyan, Karen, and Andrew Zisserman. "Very deep convolutional networks for large-scale image recognition." arXiv preprint arXiv:1409.1556 (2014).

43. Zeiler, Matthew D., and Rob Fergus. "Visualizing and understanding convolutional networks." In European conference on computer vision, pp. 818-833. Springer, Cham, 2014.

44. Simonyan, Karen, and Andrew Zisserman. "Very deep convolutional networks for large-scale image recognition." arXiv preprint arXiv:1409.1556 (2014).

45. DREAM. The digital mammography dream challenge. [Online]. Available https://www.synapse.org/Digital_Mammography_DREAM_challenge . [Assessed on 02-07-2019]

46. Hologic. Understanding ImageChecker® CAD 10.0 User Guide MAN-03682 Rev002 (2017).

47. Hupse, Rianne, Maurice Samulski, Marc Lobbes, Ard Den Heeten, Mechli W. Imhof-Tas, David Beijerinck, Ruud Pijnappel, Carla Boetes, and Nico Karssemeijer. "Standalone computer-aided detection compared to radiologists' performance for the detection of mammographic masses." European radiology 23, no. 1 (2013): 93-100.

48. Krizhevsky, Alex, Ilya Sutskever, and Geoffrey E. Hinton. "Imagenet classification with deep convolutional neural networks." In Advances in neural information processing systems, pp. 1097-1105. 2012.

49. Moreira, Inês C., Igor Amaral, Inês Domingues, António Cardoso, Maria Joao Cardoso, and Jaime S. Cardoso. "Inbreast: toward a full-field digital mammographic database." Academic radiology 19, no. 2 (2012): 236-248.

50. Krizhevsky, Alex, Ilya Sutskever, and Geoffrey E. Hinton. "Imagenet classification with deep convolutional neural networks." In Advances in neural information processing systems, pp. 1097-1105. 2012.

51. Lopez, MA Guevara, N. Posada, Daniel C. Moura, Raúl Ramos Pollán, José M. Franco Valiente, César Suárez Ortega, M. Solar et al. "BCDR: a breast cancer digital repository." In 15th International conference on experimental mechanics. 2012.

52. Jamieson, Andrew R., Karen Drukker, and Maryellen L. Giger. "Breast image feature learning with adaptive deconvolutional networks." In Medical Imaging 2012: Computer-Aided Diagnosis, vol. 8315, p. 831506. International Society for Optics and Photonics, 2012.

53. Hubbard, Rebecca A., Karla Kerlikowske, Chris I. Flowers, Bonnie C. Yankaskas, Weiwei Zhu, and Diana L. Miglioretti. "Cumulative probability of false-positive recall or biopsy recommendation after 10 years of screening mammography: a cohort study." Annals of internal medicine 155, no. 8 (2011): 481-492.

54. Fenton, Joshua J., Linn Abraham, Stephen H. Taplin, Berta M. Geller, Patricia A. Carney, Carl D’Orsi, Joann G. Elmore, William E. Barlow, and Breast Cancer Surveillance Consortium. "Effectiveness of computer-aided detection in community mammography practice." Journal of the National Cancer institute 103, no. 15 (2011): 1152-1161.

55. Eurostat. Health Statistics: Atlas on Mortality in the European Union; Office for Official Publications of the European Union: Luxembourg, 2009.

56. Hupse, Rianne, and Nico Karssemeijer. "Use of normal tissue context in computer-aided detection of masses in mammograms." IEEE Transactions on Medical Imaging 28, no. 12 (2009): 2033-2041.
57. Sadaf, Arifa, Pavel Crystal, Anabel Scaranelo, and Thomas Helbich "Performance of computer-aided detection applied to full-field digital mammography in detection of breast cancers." European journal of radiology 77, no. 3 (2011): 457-461.

58. Gilbert, Fiona J., Susan M. Astley, Maureen GC Gillan, Olorunsola F. Agbaje, Matthew G. Wallis, Jonathan James, Caroline RM Boggis, and Stephen W. Duffy. "Single reading with computer-aided detection for screening mammography." New England Journal of Medicine 359, no. 16 (2008): 1675-1684.

59. Oliveira, Júlia EE, Mark O. Gueld, Arnaldo de A. Araújo, Bastian Ott, and Thomas M. Deserno. "Toward a standard reference database for computer-aided mammography." In Medical Imaging 2008 Computer-Aided Diagnosis, vol. 6915, p. 69151Y. International Society for Optics and Photonics, 2008.

60. R. Gonzalez, R. Woods, Digital Image Processing, 3rd edition, Addison-WesleyLongman Publishing, 2008. 